\title{
El servicio desde una perspectiva integral en las organizaciones
}

Fecha de recepción: 25 de noviembre de 2010

Fecha de aprobación: 19 de mayo de 2011

\author{
Claudia Milena Manjarrez \\ cmilenam@hotmail.com \\ Politécnico Grancolombiano
}

Magíster en Gestión de Organizaciones, especialista en Alta Gerencia, profesional en Administración de Empresas y tecnóloga en Administración Hotelera; con siete años de experiencia directiva en empresas reconocidas del sector, a nivel nacional e internacional, y siete años de experiencia docente en la modalidad presencial y en ambientes virtuales de aprendizaje. Actualmente, es docente de la Escuela de Turismo y tutora virtual del programa de Administración en el Politécnico Grancolombiano.

\section{Resumen}

El servicio al cliente no es sólo el momento en el que el cliente tiene contacto con la organización. Todas las actividades que se viven en el proceso productivo deben ir enfocadas a satisfacer sus necesidades y expectativas, dando como resultado clientes satisfechos y el crecimiento y sostenibilidad de la organización en el tiempo.

\section{Palabras clave}

Organización, servicio, atención, cliente, concepto de valor, momentos de verdad, personal de servicio primario.

\begin{abstract}
Customer service is not just the time when the customer contacts the organization. All activities that are experienced within the production process must be focused on meeting their needs and expectations, resulting in satisfied customers and organization growth and sustainability over time.
\end{abstract}

\section{Keywords}

Organization, Service, Care, Customer, Value Concept, Moments of Truth, Primary Service Personnel. 
Comenzaré con los siguientes contenidos que plantea Bernardo Kliksberg sobre la definición de organización. En el contexto de los tiempos en que vivimos en la actualidad, el autor la define de la siguiente manera:

Es una institución social. Es centro de esa institución social, [sic] un sistema de actividades desempeñado por sus integrantes. El sistema de actividades se caracteriza por su coordinación consciente y su racionalidad, y crea expectativas fijas de comportamiento reciproco entre los miembros de la organización. El conjunto de relaciones entre las actividades de la organización constituyen su estructura. La estructura es de carácter relativamente estable en el tiempo. Tiende hacia determinados fines (1995).

Analizando un poco lo anterior, se puede decir que una organización está compuesta por seres humanos regulados mediante costumbres y una cultura característica, lo que la hace social; es un colectivo de personas que desempeñan diversas funciones, procesos y procedimientos que conllevan a que se evidencie su estructura organizacional. Todo esto en busca de un objetivo, que es diseñado inicialmente dentro de la planeación estratégica.

Para puntualizar el concepto macro de este artículo, voy a compartir la definición que nos da el diccionario de la Real Academia Española, que a mi entender es resumida y muy concisa: "Asociación de personas regulada por un conjunto de normas en función de determinados fines".
Analizando a nivel empresarial y organizacional, todo esto se puede explicar en el concepto de cadena productiva, que expresa que todas las áreas y actividades de la organización están enfocadas a lograr un objetivo organizacional. Generalmente, el objetivo es la obtención de utilidades. Esas utilidades son posibles si se cuenta con clientes que estén dispuestos a pagar el valor de los productos y/o servicios que ofrece la organización.

Muchas empresas recargan gran porcentaje de esa responsabilidad al personal de servicio primario, que son los que tienen contacto directo con el cliente, ya que hacen parte de los procesos productivos (llamados también centros de producción), pues allí es donde culmina todo el proceso y se genera la venta. El personal de servicio secundario, los que sirven al cliente sin ser vistos y que también hacen parte del centro de producción, y el personal de soporte, que son las demás personas de la compañía que conforman el centro de servicios de apoyo, también son responsables de que se logre este objetivo. En la gráfica 1 se pueden visualizar mejor estos conceptos.

Cuando se habla de servicio en las organizaciones, generalmente suele confundirse con el término atención al cliente, que puede definirse como el contacto interpersonal que tiene la organización con una persona, sea externa o interna, en el que ésta, llamada cliente, valora su calidad. Es una pequeña fracción de todo el proceso, pero ¿cómo se puede definir servicio? El servicio, visto desde una perspectiva integral, puede determinarse como el conjunto de actividades y/o acciones de toda la organización enfocadas en brindar bienes tangibles o intangibles que logren superar las expectativas de los clientes externos. Esta superación 
de expectativas deberá entenderse como el conocido concepto de valor, es decir, como la relación entre lo que el cliente recibe y los costos que se incurren durante todo el proceso productivo. En pocas palabras, que el cliente sienta que está pagando un valor justo o por debajo, comparado con el servicio que recibió.

Gráfica 1. Concepto de servicio al cliente.

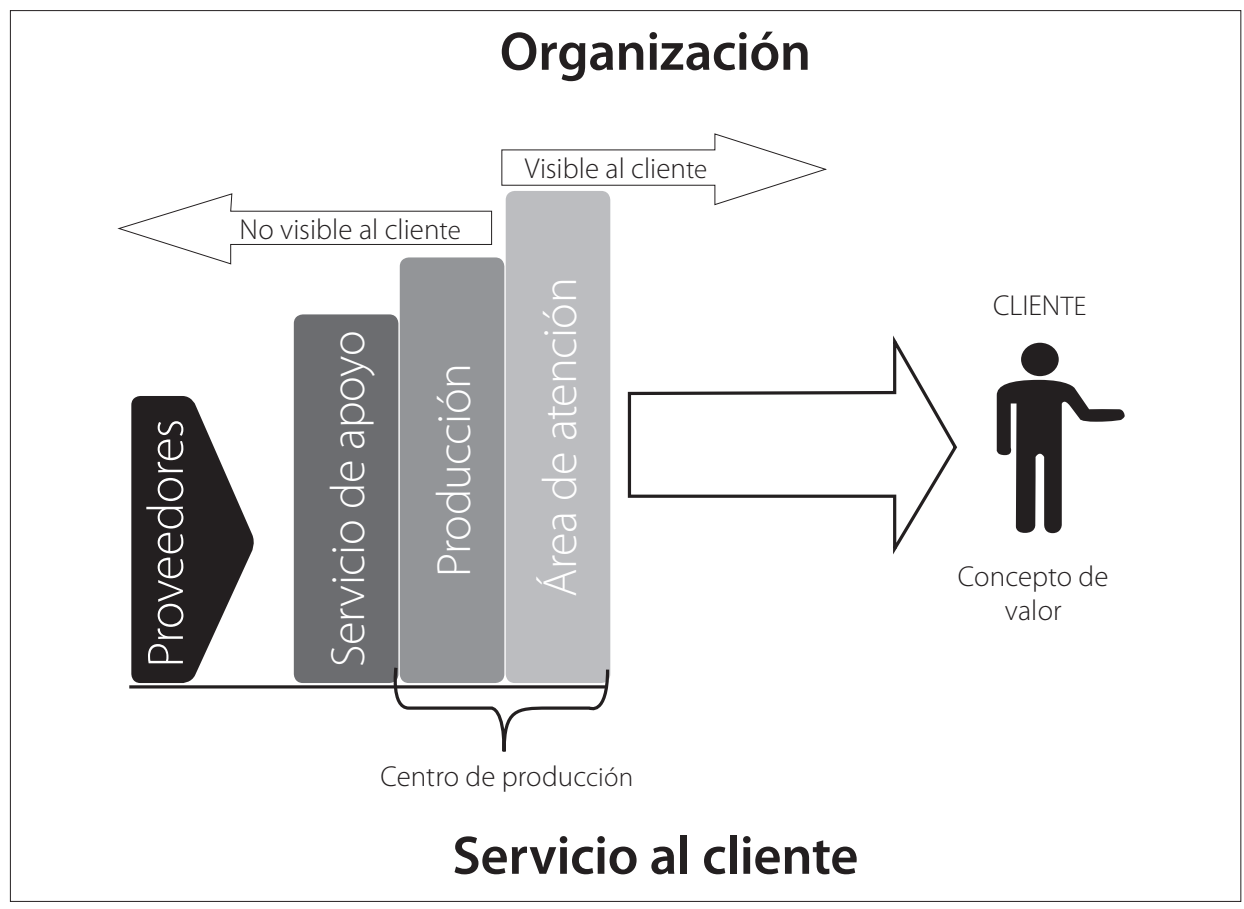

Fuente: autora.

En la gráfica 1 se explica el concepto de servicio, que se ampliará a continuación. La gráfica consta de cuatro elementos importantes, a saber: proveedores, servicios de apoyo, producción y área de atención.

\section{Proveedores}

La Real Academia Española lo define como "Persona o empresa que provee o abastece de todo lo necesario para un fin a grandes grupos, asociaciones, comunidades, etc.".

A nivel organizacional, son empresas externas a la organización que representan el inicio del proceso productivo. Su importancia se fundamenta en que si se genera una relación de mutuo beneficio, ambas organizaciones, tanto la nuestra como la del proveedor, aumentan la capacidad de las dos para crear valor.

Los proveedores son aliados estratégicos que abastecen a la organización de las materias primas o insumos con las características de calidad, precio, cantidad, 
tamaño y especificaciones, entre otras, ya previamente estandarizadas, las cuales son necesarias para comenzar un proceso con altos estándares de servicio.

Anteriormente, los proveedores estaban en un segundo plano, pero a medida que el cliente exigía cada vez más, las organizaciones, en su búsqueda constante, comenzaron a darle la importancia que en realidad tienen, trabajando mancomunadamente con ellos para lograr un producto terminado acorde al concepto de valor que tienen dentro de la promesa de venta.

El trabajo en equipo con ellos consiste básicamente en hacer trazabilidad de los productos que van a vender a la organización, confirmar procesos de calidad y disponibilidad de existencias y realizar negociaciones de precio, tiempos de entrega y formas de pago, entre otras. Evidentemente, este proceso es esencial en el concepto de valor que el cliente final percibirá en el momento que adquiera el producto y/o servicio.

\section{Centros de servicio de apoyo}

Estas áreas de la organización no tienen contacto directo con los clientes, pero realizan actividades fundamentales que dan soporte a todo el proceso productivo. Algunas son:

\section{Compras}

$\mathrm{Su}$ objetivo principal es adquirir de los proveedores los productos necesarios para satisfacer las necesidades de la organización, teniendo en cuenta, entre otros, las cantidades, especificaciones y el mejor precio y tiempo de entrega.

\section{Recepción}

A esta actividad a veces no se le da la importancia que realmente tiene y es en este punto donde se verifica si lo que se compró realmente cumple con la calidad requerida por la organización. Calidad que le permitirá cumplir con su estándar de producto y/o servicio.

\section{Almacén}

El objetivo principal de esta área es velar por la debida conservación de todas las mercancías recibidas en el proceso anterior. Un mal manejo de este proceso puede incurrir en deterioro de materias primas o pérdidas por roturas o robos y, por ende, afectar directamente los costos de la operación y la calidad del producto final.

\section{Administración y finanzas}

Orientan todos los procesos hacia el logro de los objetivos de la organización. Entre las funciones que realizan están presupuestar, ejecutar y controlar la obtención de metas.

\section{Personal}

Esta área de la organización es fundamental como apoyo a los demás procesos, en especial la selección de personal, en donde se debe tener muchísimo cuidado, considerando que no todas las personas cuentan con actitudes para ser seleccionadas como personal de servicio primario. Este es, a mi parecer, uno de los grandes errores que se cometen en el proceso de la gestión del talento humano dentro de las organizaciones, ya que se realiza un excelente trabajo seleccionando a personas muy buenas técnicamente, pero que no son competentes para estar en contacto directo con los clientes.

Según Ivancevich, "selección es el proceso mediante el cual una organización 
elige, entre una lista de candidatos, la persona que satisface mejor los criterios exigidos para ocupar el cargo disponible, considerando las actuales condiciones de mercado" (1995). Hoy en día, la selección se debe basar en las competencias laborales, tomando como referencia que en todas las organizaciones se requieren personas de servicio primario (que están en contacto directo con los clientes externos), lo que significa que deben tener dominio en un área técnica y poseer valores adicionales que las hagan realmente competentes.

Se define competencia laboral a "la capacidad de una persona para desempeñar una función productiva, en contextos variables y con base en estándares de calidad establecidos por el sector productivo, que requiere la interacción de conocimientos, habilidades, comprensión y valores" (SENA, 2004).

Hago énfasis en este aspecto porque se cree que la persona con capacitaciones puede mejorar su actitud frente al cliente, pero lo que realmente puede mejorar es su aptitud para su puesto de trabajo. Seguramente se logrará que la persona aprenda algunas técnicas, como saludar y sonreír, pero si eso no está intrínseco dentro del ser humano, rápidamente lo dejará de poner en práctica.

\section{Producción}

Este proceso hace parte de los centros de producción. Aunque es un área que la mayoría de las veces no tiene contacto directo con el cliente, es donde se transforman todas las materias primas e insumos en el producto terminado que el cliente ha solicitado o va a comprar. Es aquí donde se debe contar con altos estándares de calidad. Se deben tener normalizados todos los procesos y procedimientos, tanto de los productos como de cada una de las funciones del área.

Todos, generalmente, somos usuarios de establecimientos gastronómicos, sea por necesidad o buscando esparcimiento. Por esta razón, voy a tomar como ejemplo este tipo de organización para describir detalladamente lo que debe tenerse en cuenta en esta área de producción.

El personal trabajador del centro de producción de un establecimiento gastronómico debe tener capacitaciones específicas en buenas prácticas de manufactura, puntos críticos de control y elaboración de recetas, siguiendo los manuales de productos. Debe contar con una excelente presentación personal e higiene, que comprenden uniformes limpios y en perfecto estado, siguiendo la normatividad exigida por los entes de control, calzado adecuado, cabello recogido y limpio, uñas cortas y evitar el uso de maquillaje, joyería y bisutería, entre otros. Por su parte, los equipos y utensilios también deben cumplir requisitos básicos que garanticen que el producto final va a llegar a la mesa del cliente con los más altos estándares de higiene y calidad. Además, la infraestructura de la zona debe estar diseñada de acuerdo al decreto de manipulación de alimentos que rige el ente de control.

\section{Área de atención}

En el área de atención se concentran todos los procesos que se desarrollan en contacto directo con el cliente. En este punto de nuestra cadena de valor es 
donde se evidencia si el proceso de la gestión de talento humano funciona efectivamente y se han seleccionado trabajadores no sólo con aptitudes, sino con actitudes de servicio, y si las cosas se están haciendo bien en cada uno de los procesos de la organización, lo que implica la comprobación de contar con una cultura de servicio bien estructurada.
Aquí es donde empezamos a ver conceptos clásicos de servicio, como los momentos de verdad, que, según Karl Albrecht, son definidos por la gerencia de servicio de la siguiente manera: "un episodio en el cual el cliente entra en contacto con cualquier aspecto de la organización y tiene una impresión sobre la calidad de su servicio" (2006).

Gráfica 2. Perspectiva integral de los momentos de verdad.

\begin{tabular}{|c|c|}
\hline $\begin{array}{l}\text { - Expecativas } \\
\text { - Necesidades } \\
\text { - Dinero } \\
\text { - Curiosidad }\end{array}$ & $\begin{array}{l}\text { Concepto de valor } \\
\text { - Satisfacción } \\
\text { - Sentimientos positivos o } \\
\text { negativos } \\
\text { - Desilusión }\end{array}$ \\
\hline Entradas & Salidas \\
\hline $\begin{array}{l}\text { - Cultura } \\
\text { - Materias primas } \\
\text { - Procesos } \\
\text { - Actitudes } \\
\text { - Aptitudes } \\
\text { - Infraestructura } \\
\text { - Normas } \\
\text { - Equipos } \\
\text { - Selección idónea } \\
\text { - Capacitación }\end{array}$ & $\begin{array}{l}\text { - Crecimiento en ventas } \\
\text { - Pérdida de clientes } \\
\text { - Pérdida de dinero }\end{array}$ \\
\hline
\end{tabular}

Fuente: autora.

En la gráfica 2 vemos de manera integral la explicación de los momentos de verdad. Una de las características de los momentos de verdad es que son del cliente, y es por eso que su análisis debe hacerse desde la perspectiva de éste.

También se aprecia en esta gráfica que los momentos de verdad tienen unas entradas y unas salidas. El cliente contacta a una organización porque tiene necesidades, expectativas, curiosidad y dinero para pagar un bien tangible o intangible que ella ofrece. Se presenta, así, el momento de verdad que dará como resultado el concepto de valor que puede ser satisfacción de su expectativa, sentimientos de alegría o desilusión, entre otros.

Es integral porque para lograr la salida de los momentos de verdad del cliente, la organización también tiene unas entradas, como son materias primas e insumos, procesos, infraestructura y equipos. Todo fundamentado en una cultura de servicio que, una vez generados los 
momentos de verdad de los clientes, va a dar como salida crecimiento en ventas o pérdida de dinero, entre otras.

Retomando el ejemplo del restaurante, la infraestructura de esta área también está reglamentada y debe cumplir con especificaciones de materiales, ventilación y servicios sanitarios, entre otros.

En cuanto a las especificaciones físicas generales, hay que tener presente que la primera impresión es una de las más importantes, por eso el exterior del establecimiento debe informar a los clientes qué tipo de establecimiento han elegido, y debe seguir el reglamento de sanidad e higiene que exige el decreto 3075 que, para el caso colombiano, expidió el Ministerio de Protección Social.

Con respecto al restaurante, a continuación describiré aspectos fundamentales para la generación de momentos de verdad.

- Contar con un aviso llamativo que dé una correcta descripción e impresión de la calidad que va a encontrarse dentro del establecimiento.

- La exhibición del menú debe estar publicada acorde a la ambientación del establecimiento.

- Es importante establecer un tema o estilo para que la ambientación, los menús, los uniformes y todo lo relacionado al establecimiento tengan una clara identidad.

- La temperatura ideal dentro del salón comedor es de $18^{\circ} \mathrm{C}$.

- Tener señalizados los diferentes espacios dentro del establecimiento, como son servicios de baños para damas, servicios de bańos para caballeros, sistemas de extinción de incendios y salidas de emergencia, entre otros.
- Uno de los aspectos que aporta mucho valor a los clientes es el uniforme y la presentación personal en las áreas de atención. Por eso me permito realizar una breve descripción de este:

- Uniforme, en perfecto estado de limpieza y planchado.

- Para las damas, cabello totalmente limpio. En caso de tenerlo largo, la malla debe recogerlo todo dentro de la mońa; para el cabello corto, cubrirlo todo con una malla invisible.

- Para los caballeros, corte estilo militar y afeitados.

- Portar siempre la identificación o carné de la organización.

- Únicamente permitir la utilización de aretes estilo topito en el caso de las damas.

- Utilizar maquillaje muy suave (obviamente para las damas).

- No usar perfumes, pues se puede pasar a los alimentos.

- Uńas cortas y sin ningún tipo de maquillaje.

- Mantener dos limpiones en perfecto estado de limpieza y diferenciados. Uno para limpiar las sillas y el otro para limpiar las mesas.

- El comportamiento del personal de esta área debe estar marcado por actitudes de servicio que casi siempre son intrínsecos de las personas, y por conocimientos que se pueden adquirir mediante capacitaciones, como por ejemplo:

- Protocolos de servicio.

- Normas de etiquetas.

- Conocimiento de las preparaciones e ingredientes de cada uno de 
los componentes de la carta para poder brindar una asesoría honesta a los comensales.

- Capacitación como manipuladores de alimentos.

- Conocimiento de rutinas de higiene para equipos, muebles y enseres de las áreas de servicio.

- Saber cómo actuar en caso de quejas, reclamos y felicitaciones.

\section{Concepto de valor}

El cliente, al igual que el mundo y las organizaciones, también cambia, convirtiéndose en una persona u organización cada vez más conocedora y exigente. Es por esto que selecciona la empresa que les ofrezca mayor valor por su compra.

El diccionario de la Real Academia Española define valor como "Grado de utilidad o aptitud de las cosas, para satisfacer las necesidades o proporcionar bienestar o deleite". Una segunda definición que plantea el diccionario es "Cualidad de las cosas, en virtud de la cual se da por poseerlas, cierta suma de dinero o equivalente". A su vez, M. Porter lo define de la siguiente manera: "valor es la cantidad de dinero que los clientes están dispuestos a pagar por los productos o servicios de la empresa" (Porter, 1985).

Partiendo de los anteriores conceptos, el cliente tiene unos determinantes que aumentan o disminuyen el valor y su satisfacción con una organización. Siguiendo con el ejemplo del establecimiento gastronómico, las personas no vamos a un establecimiento sólo a suplir una necesidad fisiológica, también lo hacemos para buscar un conjunto de elementos que nos brindan satisfacción. Algunos de estos elementos son:
- Presentación del establecimiento: aseo, mantenimiento, olor, ambientación y otros.

- Atención del personal de servicio: saludos, protocolos, normas de etiqueta, presentación personal, higiene del personal, conocimiento del producto y cortesía, entre otros.

- Calidad del plato: aroma, presentación de los alimentos en el plato, colores, textura, balance nutricional, temperatura y sabor, entre otros.

- Infraestructura: comodidad de los muebles, vajilla, cubertería, áreas seguras y delimitadas, higiene y otros.

- Atención: tiempo de respuesta, cordialidad en el trato, actitud de servicio, tolerancia, preocupación, asesoría y otros.

- Precio: el precio debe ser equivalente a todo lo anterior; que el cliente sienta que paga lo justo por lo recibido en su decisión de compra.

Como se puede apreciar en este artículo, un sólo aspecto que falle en la cadena productiva de una organización podrá generar insatisfacción en el cliente. Claro está que con una excelente atención por parte del personal de servicios primarios (contacto directo con los clientes), que es casi siempre la única área visible para los clientes externos, se puede disimular o disminuir una experiencia negativa. No todos los trabajadores tienen competencias para atender personas, por eso es clave tener esto en cuenta al momento de realizar los procesos de selección del personal de servicio primario en todas las organizaciones.

Deseo concluir que para lograr excelencia en el servicio hay que hacer las cosas bien en todas las áreas y procesos de la organización. Cada una de las 
decisiones y actuaciones dentro de la organización va a repercutir en la satisfacción o insatisfacción del cliente y, como resultado operacional, en las utilidades o pérdidas organizacionales. "Estamos tan acostumbrados al mal servicio que, cuando nos brindan un nivel de servicio normal, nos sentimos felices" (la autora).

\section{Referencias}

I. Albrecht, K. (2006). La revolución del servicio. Bogotá: Panamericana Editorial Ltda.

2. Chiavenato, I. (2002). Gestión del talento humano. Bogotá: McGraw-Hill.

3. Covey, S.R. (1984). Los siete hábitos de la gente eficaz. Barcelona: Ediciones Paidós IbéricaDahmer, S. \& Kahl, K. (2002). Restaurantes: Servicio básico. Zaragoza: Editorial Acribia, S.A.

4. García, J. \& Manga, M. (2007). Inteligencia relacional. Bogotá: Javier Vergara Editor.

5. Goleman, D. (1996). La inteligencia emocional. Buenos Aires: Javier Vergara Editor.

6. Hall, R. (1996). Organizaciones: estructuras, procesos, resultados. México: Prentice Hall.

7. Ivancevich, J.M. (1995). Human Resource Management. Nueva York: Richard D. Irwin.

8. Kliksberg, B. (1995). El pensamiento organizativo: de los dogmas al nuevo paradigma gerencial (13a ed.). Buenos Aires: Tesis-Norma.
9. Lelan, K. \& Bailey K. (1995). Servicio al cliente para dummies. California: Grupo Editorial Norma.

ıo.Maqueda Lafuente, J. \& Llaguno Musons, J.I. (1995). Marketing estratégico para las empresas de servicio. España: Ediciones Días Santos.

ir.Manjarrez Alzate, C.M. (2007). Modelo gerencial para microempresas del sector gastronómico. Tesis de grado obtenido no publicada. Universidad Militar Nueva Granada. Bogotá, Colombia.

I 2.Paz Couso, R. (2010). Atención al cliente. España: Editorial Ideas Propias.

I3.Prieto Herrera, J.E. (2010). Gerencia del servicio: La clave para ganar todos. Bogotá: Ecoe Ediciones.

I4.Porter, M. (1985). Competitive Advantage. New York: Free Press.

I 5. Rosenbluth, H. \& Mc Ferrin Peters, D. (1992). El cliente no es lo primero. Buenos Aires: Atlántida.

i6. Secretaría Distrital de Salud. (1997). "Decreto 3075".

I7.Serna Gómez, H. (1996). Auditoría del servicio. Bogotá: RAM Editores.

I 8. Sheel Mayenberger, A. (1992). Control de alimentos y bebidas I. Enciclopedia de hotelería y restaurantes. Bogotá: Universidad Externado de Colombia.

I9.Silverman, D. (2008). ¿Podrán salvarnos nuestros clientes? Harvard Business Review América Latina.

20.Vargas Quiñones, M.E, \& Aldana de Vega, L. (2007). Calidad y servicio conceptos y herramientas. Bogotá: Ecoe Ediciones. 